Projets

de paysage

\section{Projets de paysage}

Revue scientifique sur la conception et l'aménagement de l'espace

\section{$5 \mid 2011$ \\ Le jardin et ses rapports à l'art. Encadrer, décadrer, recadrer}

\title{
La rencontre du paysage
}

Meeting of the landscape

Joël Bouderlique

\section{(e) OpenEdition}

\section{Journals}

\section{Édition électronique}

URL : https://journals.openedition.org/paysage/21588

DOI : $10.4000 /$ paysage. 21588

ISSN : 1969-6124

\section{Éditeur :}

École nationale supérieure du paysage de Versailles-Marseille, Institut national des sciences appliquées Centre Val de Loire - École de la nature et du paysage, École nationale supérieure d'architecture et de paysage de Bordeaux, École nationale supérieure d'architecture et de paysage de Lille, Agrocampus Angers

\section{Référence électronique}

Joël Bouderlique, «La rencontre du paysage », Projets de paysage [En ligne], 5 | 2011, mis en ligne le 19 janvier 2011, consulté le 16 octobre 2021. URL : http://journals.openedition.org/paysage/21588 ; DOI : https://doi.org/10.4000/paysage.21588

Ce document a été généré automatiquement le 16 octobre 2021.

\section{c) $($ ()) $\Theta$}

La revue Projets de paysage est mise à disposition selon les termes de la Licence Creative Commons Attribution - Pas d'Utilisation Commerciale - Pas de Modification 4.0 International. 


\section{La rencontre du paysage}

Meeting of the landscape

Joël Bouderlique

Je parle avec ma main, tu écoutes avec tes yeux ; et nous nous comprenons, n'est-ce pas, en un seul

sourire.

Shitao $^{1}$

1 Deux marches de quelques semaines effectuées en 1981 dans l'Himalaya, l'une au Népal l'autre au Ladakh, ont radicalement transformé ce que j'entendais jusqu'alors dans le terme de paysage. L'année suivante deux "pèlerinages » en Chine ont accentué le contraste entre ce que je savais du paysage et ce que j'avais commencé à en connaitre. Puis, la fréquentation régulière des jardins, durant la vingtaine d'années passées ensuite au Japon, a prolongé les réflexions résumées dans ce texte qui vise à rendre possible une rencontre du paysage sauvage ou artificiel.

2 Réputés jadis comme d'effectifs pèlerinages bouddhiques, les trois jours de marche pour gravir et redescendre les monts Huang ${ }^{2}$, ainsi que les cinq jours nécessaires pour accomplir un périple identique au mont 0 mei $^{3}$, offrent aujourd'hui comme hier une richesse de paysages que de nombreux peintres ont pris pour sujet. 
Figure 1. La montagne Huang Shan

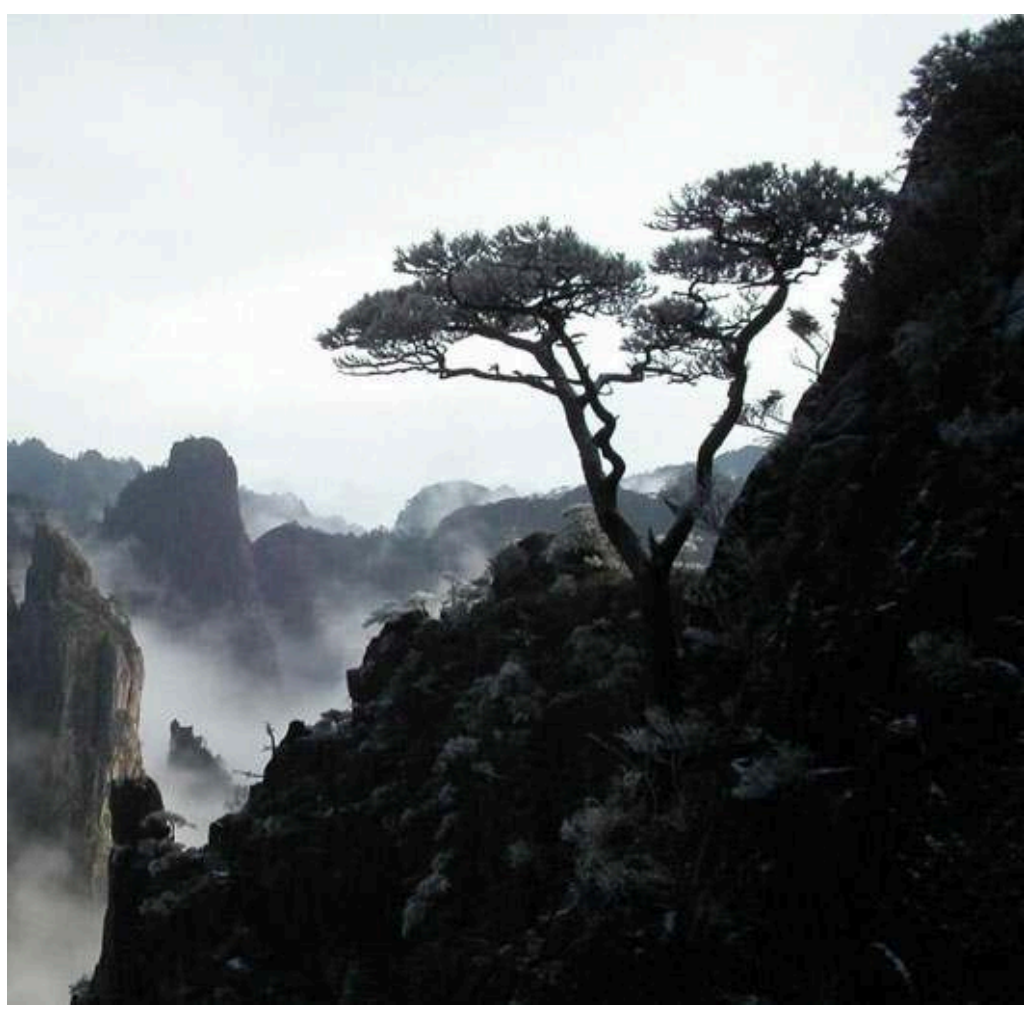

3 Certaines des œuvres picturales produites en ces lieux hors du commun sont en ellesmêmes directement évocatrices de ce que les mots vont tenter à leur façon d'exprimer : la rencontre elle-même qui se produit dans ces sites, rencontre que nous nommons : paysage.

4 Les mots, et plus particulièrement l'usage qui en est fait, sont donc déterminants dans un texte pour mettre en prise avec ces instants d'espace dont la durée fugitive persiste, en soi immuable, après tant d'années. Lors des marches elles-mêmes, le vocabulaire employé était extrêmement varié pour décrire les roches, les chutes d'eau, les arbres, les brumes qui masquent et révèlent plus ou moins les reliefs, ou le halo brumeux circulaire spécifique au lever du soleil au sommet du mont Omei. Les nombreux, très nombreux, marcheurs chinois n'étaient pas avares d'explications susceptibles de faire appréhender aux « étrangers » toute la beauté de tel rocher appelé le "singe assis avec son petit sur le dos", de tel autre désigné comme "la grenouille», de tel arbre accroché sur cette falaise nommé "le héron qui picore", ou de cette apothéose matinale de l'aurore, applaudie par quelques milliers de "pèlerins" comme "l'illumination de l'auréole du Bouddha». Toutefois, ces prolifiques explications visualisantes auraient plutôt eu tendance - peut-être faute de résonances culturelles appropriées - à constituer un catalogue d'images projectives (offert par ailleurs en de multiples déclinaisons dans les guides touristiques) qui plantent un décor imposé en face du marcheur. Ainsi se constitue ce qui se nomme une objectité, découlant précisément du procédé spéculatif objectivant de ce qu'autrement il faut bien appeler un phénomène, c'est-à-dire de ce qui apparaît. Dans le premier cas, il y a d'un côté un répertoire préconstitué d'ob-jets, soit préfabriqués par une tradition, soit produits personnellement dans cette perspective analytique objectivante qui met hors-jeu la dimension esthétique, et de l'autre un sujet séparé qui se satisfait de ses projections 
largement partagées. En revanche, dans le cas de l'apparition elle-même de ce que nous nommons le paysage, la distinction entre le sujet et le monde qui surgit est véritablement problématique, $a$ fortiori dans l'instant de l'apparaître, car cette question ne se pose pas alors : l'espace et le temps sont pleins... de cette apparition elle-même, soi et monde « $y$ » sont un-unique.

Comment ce phénomène peut-il se produire ? S'y attendre ou l'attendre serait déjà le préfaçonner, il est forcément hors d'attente. L'espérer? Mais alors de façon informelle, comme l'inespérable... qui étonne - pas celui qui l'attend - mais qui est en soi étonnement. Ce qui s'impose dans le paysage, sans division entre soi et monde, est le dévoilement de l'être, seul réel. « Le Réel c'est toujours ce que l'on n'attendait pas. Mais quand l'inattendu se produit on le découvre comme toujours déjà là. " (Maldiney, 1, 1973, p. 143.) Toutefois la puissance de cette dernière affirmation laisse dans l'incertitude quant à la nature du deuxième " on ", celui qui constate cette découverte. Lui-même ne peut pré-exister à sa découverte car la révélation qu'il fait, le fait luimême. Le premier «on » est bien là, constitué dans son attente, mais le deuxième ne vient qu'après-coup, lorsqu'il se reconstitue pour le constat ${ }^{4}$. Non seulement l'instant de la révélation du paysage est unique, mais elle est unicité. Le sujet s'y trouve (dans les deux sens du verbe : y être et s'y découvrir) aussi comme ex-istant, c'est-à-dire comme participant nécessaire à cette apparition-émergence de l'être, nommée globalement paysage. Comment cette donation est-elle possible ? L'étiquetage anticipé l'empêche, il n'y a plus donation mais reconnaissance de formes déjà attribuées : le petit singe, la tortue, le vieux sage, etc., par exemple. Le blocage volontaire de cette attitude objectivante l'entrave aussi par une pré-occupation qui occupe le terrain mental et de la sorte empêche l'ouverture. Seule la rupture provoquée par la sur-prise fait lâcher l'emprise habituelle à la thématisation du monde résultant de sa formatisation par une conceptualisation qui l'anticipe toujours. À l'inverse, lorsque surgit dans l'ouvert l'unicité du paysage, l'étonnement de sa donation donne aussi le ton de sa présence. Présence tonale qui est indissociablement celle des conditions de son intelligibilité et celle des conditions de sa possibilité formelle même. Souvent formulé au plus simple par les Japonais dans un « Ah! » expressif de l'étonnement, celui-ci peut aussi prendre forme dans une parole dont l'expression, au plus proche de son origine, est poétique. C'est là aussi que la peinture de paysage a son départ.

6 Le paysage en tant que phénomène, c'est-à-dire apparition globale qui n'est possible qu'en tant que présence jamais réductible à un savoir représentatif - celui-ci n'étant jamais en prise sur ce qui est donné -, est-il dès lors la négation de la rencontre entre un milieu et un sujet qui relèverait d'une dualité ? Oui et non! Non, parce qu'il y a bien un milieu, sauvage ou artificiel, en tant que données minérales, végétales, aquatiques, de même qu'un organisme qui le rencontre. Il y a donc bien dualité. Mais oui aussi, parce qu'en tant que paysage il - à savoir le paysage en tant que phénomène - est cette rencontre même. Il y a un saut qualitatif entre, d'une part, l'expérience du milieu que fait l'individu lorsqu'il le saisit comme une représentation dont il est le détenteur distinct et, d'autre part, la rencontre dont les termes changent leurs statuts respectifs pour les faire devenir sujet et monde, unis dans une seule présence. À proprement parler, le paysage ne peut ex-ister qu'ainsi. Il s'agit précisément entre les deux modes d'être, du passage de l'ordre représentatif à l'ordre esthétique. La différence entre les deux est identique à celle du rapport que peut avoir un cartographe avec la sainte Victoire et celui qu'avait Cézanne avec cette même montagne, rapport sans lequel la création de ses œuvres eut été impossible. Pour ceux qui ne tiennent pas à entendre 
cette différence, vaut déjà la forte parole de Charles Péguy : «Ils prennent toujours l'histoire pour l'événement, la carte pour le terrain, la géographie pour la terre. Qu'ils nous permettent de leur dire : tout cela n'est pas si simple ni si vide, ni si mort. Tout cela est là.» (Péguy cité par Charcosset et Rordorf, 1973, p. X.)

7 Pour le paysagiste, le créateur de paysage, c'est-à-dire pour celui qui cherche à rendre possible une telle rencontre d'ordre esthétique, la visée escomptée relève donc d'une démarche artistique qui exige d'être explicitée.

8 La première exigence est celle esquissée au départ de ce texte, celle du regard car regarder n'est pas simplement voir. "Dans la garde qu'il institue, le regard n'est pas seulement fixé sur la chose proche, il fait sans cesse retour sur soi pour ouvrir à nouveau l'espace sans cesse renaissant de l'événement. Compris à partir de la garde, le regard n'est pas l'exercice d'une surveillance avide de prendre les choses en flagrant délit, il est la vigilance d'une attention accordée à leur être.» (Charcosset et Rordorf , 1973, p. IX.) Et pour l'artiste il faut qu'il soit tel que Francis Ponge l'évoquait: «le regard de telle sorte qu'on le parle ", c'est-à-dire pour le paysagiste, de telle sorte qu'il le fait.

9 Pour rendre possible une rencontre qui ne soit pas celle d'un individu avec un environnement paysagé, mais celle du paysage lui-même, il faut d'abord co-naître dans son éprouvé. Cette co-naissance déploie l'espace du paysage qui est paradoxalement le lieu sans lieu de l'être perdu. Erwin Straus en fait une analyse rigoureuse ${ }^{5}$ dans laquelle il précise que dans le paysage, « l'espace m'enveloppe à partir de mon ici et je ne suis ici qu'au large de l'espace sous l'horizon duquel je suis hors. Nulles coordonnées. Nul repère. » (Maldiney, 1, 1973, p.143.) Il précise aussi : «Du paysage il n'y a pas de développement qui conduise à la géographie; nous sommes sortis du chemin; comme hommes nous nous sentons perdus ${ }^{6}$.» Erwin Straus justifie cette discontinuité par une analyse comparée des deux espaces. Nous pouvons sortir du paysage pour entrer dans la géographie mais nous y perdons notre ici : « Nous n'avons plus de lieu. Nous n'avons plus lieu.» (Ibid., p. 143.) En fait, lorsque nous passons de l'espace du sentir à celui de la perception, nous passons d'un mode d'être à un autre radicalement différent : du mode d'être du sentir au mode d'être de la perception, c'est-à-dire de celui de la co-naissance à celui de la connaissance ${ }^{7}$. Invisible à la connaissance géographique, chaque éveil propre à la rencontre du paysage rend visible « le surgissement dans l'Ouvert qui révèle l'ouverture comme l'abîme de notre ancienne perdition et de notre nouvelle exposition à l'être » (ibid., p. 144).

10 L'espace du sentir, propre à la dimension du paysage, est celui du monde initial prélinguistique dans lequel chaque sentir est un ressentir ; ressentir vécu dans un espace pathiquement orienté selon les directions opposées du sympathique et de l'antipathique qui correspondent à l'ouverture et à la fermeture de la présence. Espace tissé de zones de lumière et de zones d'ombre qui offrent des directions de sens (Binswanger) associées aux mouvements de la présence. L'articulation polaire du proche et du lointain, familière à l'espace de la représentation, se trouve transformée dans chaque convocation de l'invisible propre à la rencontre de paysages naturels et artistiques, et avec elle est mise aussi en question la structure fondamentale de l'espace et du temps de la connaissance. Lors de la rencontre du paysage, la tendance essentielle de la présence à la proximité étudiée par Heidegger ${ }^{8}$ se déploie à l'écart de la zone moyenne dans laquelle s'inscrit la perspective de la représentation, pour conjuguer la tension entre proche et lointain au point de les éprouver comme des absolus qui 
s'identifient. Ainsi en est-il au franchissement d'un col au Népal lorsque dans l'ouvert du ciel se produit soudain dans le proche absolu le surgissement de l'inaccessible sommet du Dhaulagiri'.

Figure 2. Le sommet du Dhaulagiri

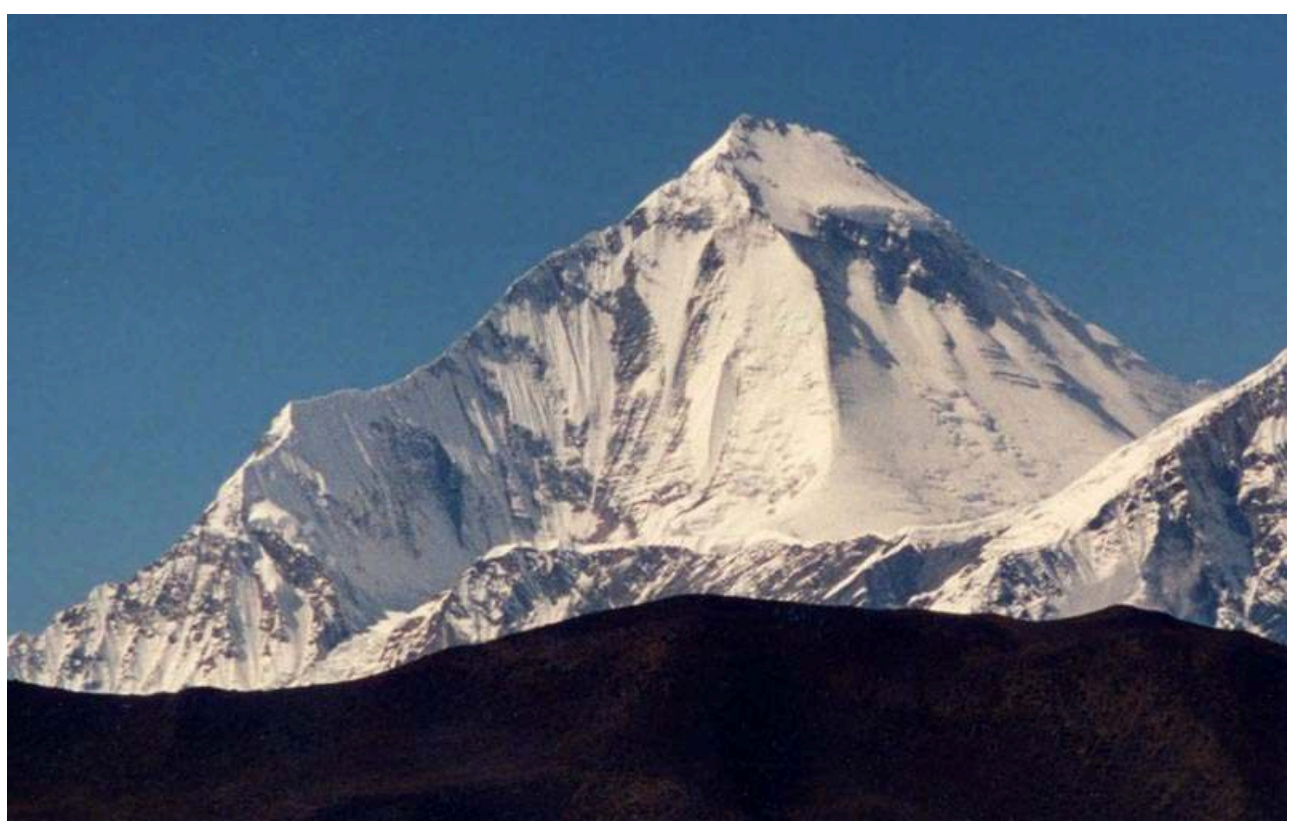

« Nulle proximité n'approche celle d'un lointain en suspens. Elle est proche parce que dans l'ouvert. Surgie de l'abîme, comme "les terres rouges" dont parle Cézanne. » (Maldiney, 1, 1973, p. 145.)

11 Le moment esthétique de la rencontre du paysage n'est pourtant pas celui d'un abandon à l'immédiat ni celui d'une régression fusionnelle dans lesquels notre pouvoirêtre démissionnerait de lui-même. Il est le surgissement qui « de l'être perdu dans le paysage fait un être en étonnement dans l'ouvert» (ibid., p.146) où s'éveille sa transcendance.

La société japonaise exige une étiquette étudiée dans tous les rapports sociaux, y compris dans ceux internes à la famille où là aussi les jeux de rôles sont fermement établis et rappelés dans les appellations de chacun et les formes verbales hiérarchisées qui les spécifient. Ces identités sociales, familiales, voire « amicales » relèvent de ce que l'on nomme en japonais le tatemae (qui se prononce taté maé), littéralement « ce qui est construit devant » et que l'on traduit un peu hâtivement par la face. Il demeure que derrière cette face "publique » il y a ce qui est nommé en japonais le honne (qui se prononce hon'né) littéralement "la racine authentique », qui demeurera cachée ou qui ne sera en tout cas que très rarement exprimée. Serait-ce en raison de cette particularité normative que les mondes des arts, seuls à offrir des moments d'authenticité, sont si largement prisés et pratiqués dans cette culture ? L'appréciation des paysages porte à le croire et l'art paysager, pratiqué de façon personnelle chez soi ${ }^{10}$ ou partagée dans les jardins d'accès public, tend à le confirmer.

13 Les jardins japonais ${ }^{11}$ peuvent grossièrement se diviser en deux groupes : ceux qui sont conçus en fonction de l'arrière-plan naturel et ceux qui appartiennent à un espace enclos. 
Figure 3. La villa impériale Shugaku-in

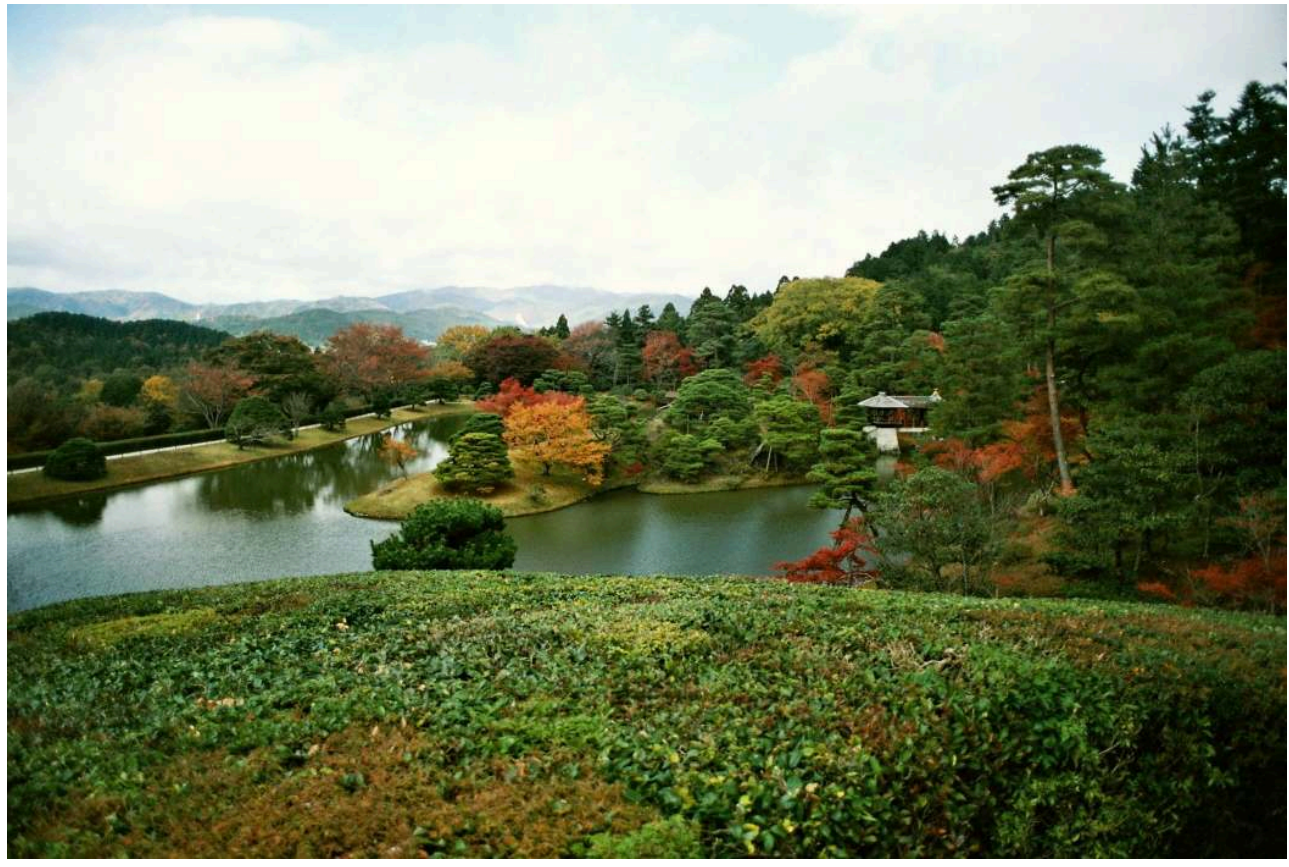

Figure 4. La villa impériale Katsura

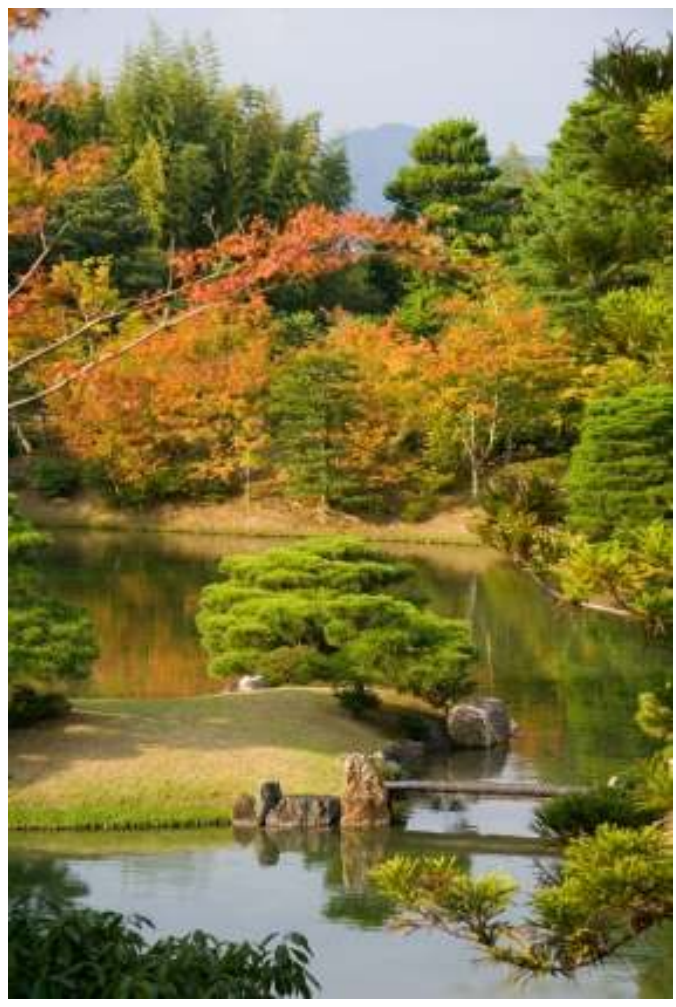


Figure 5. La villa impériale Katsura

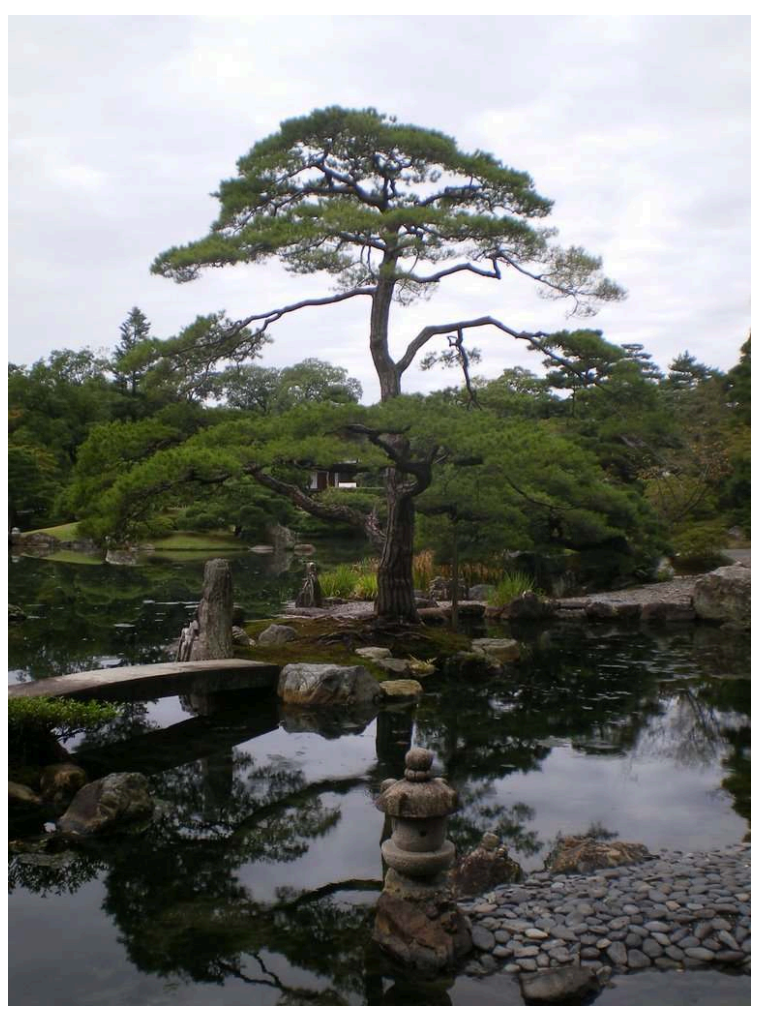

Fréquenter les uns ou les autres provoque généralement le même ressentir propre à la rencontre du paysage. Les Japonais les considèrent comme des lieux d'être et des lieux de séjour, ces deux formulations étant regroupées en langue japonaise par un seul terme : $i b a s h o^{12}$.

Dans les jardins réalisés en relation avec l'arrière-plan, c'est précisément cette relation qui en dicte la composition afin d'entrelacer dans les formes créées la zone proche et la zone lointaine. Conçus comme des parcours, ils offrent, lors des déplacements auxquels ils engagent par d'étroits sentiers, des sur-prises: une colline, objectivement dans le lointain, mais dont la base est masquée d'ici par un avant-plan végétal ou minéral dans le jardin, apparaît soudain à un point du parcours suspendu dans une proximité qui dément la distance géométrique. Une halte suggérée par la plateforme d'un pavillon de thé laisse contemplatif dans la relation rythmique entre la forêt lointaine et son reflet à la surface de l'étang situé juste au pied de cette terrasse. L'infranchissable étendue d'eau peut se parcourir pas à pas en sautant de pierre en pierre à la surface de son fond obscur peuplé de carpes multicolores. Le murmure du petit ruisseau que l'on approche devance la silencieuse cascade au loin. Le bruissement du feuillage des bambous fait écho au plus près aux remous du vent dans la lointaine forêt. La complexité des parcours impose des variations rythmiques de déplacement qui mettent en tensions harmonieuses ou contradictoires le mouvement et le regard. Regard qui glisse souplement, par exemple durant une pause, entre la proximité des éléments du jardin et le mouvement d'approche de ce lointain qu'exige la présence, ou bien, lorsque, en raison de l'étroitesse des supports, il faut sauter d'un pied sur l'autre, et qu'il s'enfonce dans les profondeurs d'une mare tout en étant contrarié par les fleurs de nénuphars qui l'attirent à sa surface. 
Dans les jardins enclos, les variations spatialisantes sont provoquées dans le monde propre qu'ils constituent et éventuellement accentuées ou atténuées par les variations temporelles sonores de ruissellements, de clapotis, ou les percussions en apparence régulières d'un marteau à eau formé d'une section de bambou, montée sur un axe, se remplissant plus ou moins rapidement d'eau jusqu'à son basculement qui provoque la frappe sur la pierre-enclume. Participent également à ces variations rythmiques les bruissements, craquements, cliquetis des feuillages selon les essences choisies. La pluie n'est pas exclue de la composition des jardins, bien au contraire au Japon il ne s'agit pas de la pluie mais des pluies dont la variété est notée par un vocabulaire très riche pour expliciter les divers éprouvés qu'elles engendrent, de même que pour la neige, dont la diversité est aussi consciencieusement répertoriée dans de riches glossaires ${ }^{13}$. Selon les météores, le mode de rencontre change, la résonance entre milieu et organisme provoque des ambiances climatiques qui correspondent à des tonalités affectives changeantes ${ }^{14}$. À chaque moment le promeneur en tant qu'être perdu ressent la variété du milieu où il se trouve - et se trouve lui-même, se retrouve - provisoirement. Là encore, comme dans la peinture de paysage pour Erwin Straus et plus généralement pour l'art entier selon Paul Klee, il ne s'agissait pas de représenter ce que nous voyons d'habitude, mais de rendre visible l'invisible, ici la présence originaire d'un promeneur perdu et ouvert à l'étendue close qui s'ouvre à lui selon les divers modes de la pluie et du beau temps, lors de ses déplacements dans le jardin. À chaque instant de son cheminement dans ces jardins, le visiteur est enveloppé sous un horizon déterminé par son ici et il n'est ici que dans l'ouverture de l'espace «sous l'horizon duquel il est présent à tout, et partout hors de lui » (Maldiney, 2, 1973, p. 150).

17 Le cas particulier des jardins zen de pierre ou de sable ${ }^{15}$ offre des possibilités de rencontres de paysages particulièrement abruptes et en cela d'autant révélatrices du procès en jeu. 
Figure 6. Le temple du Pavillon d'argent, le Ginkaku-ji

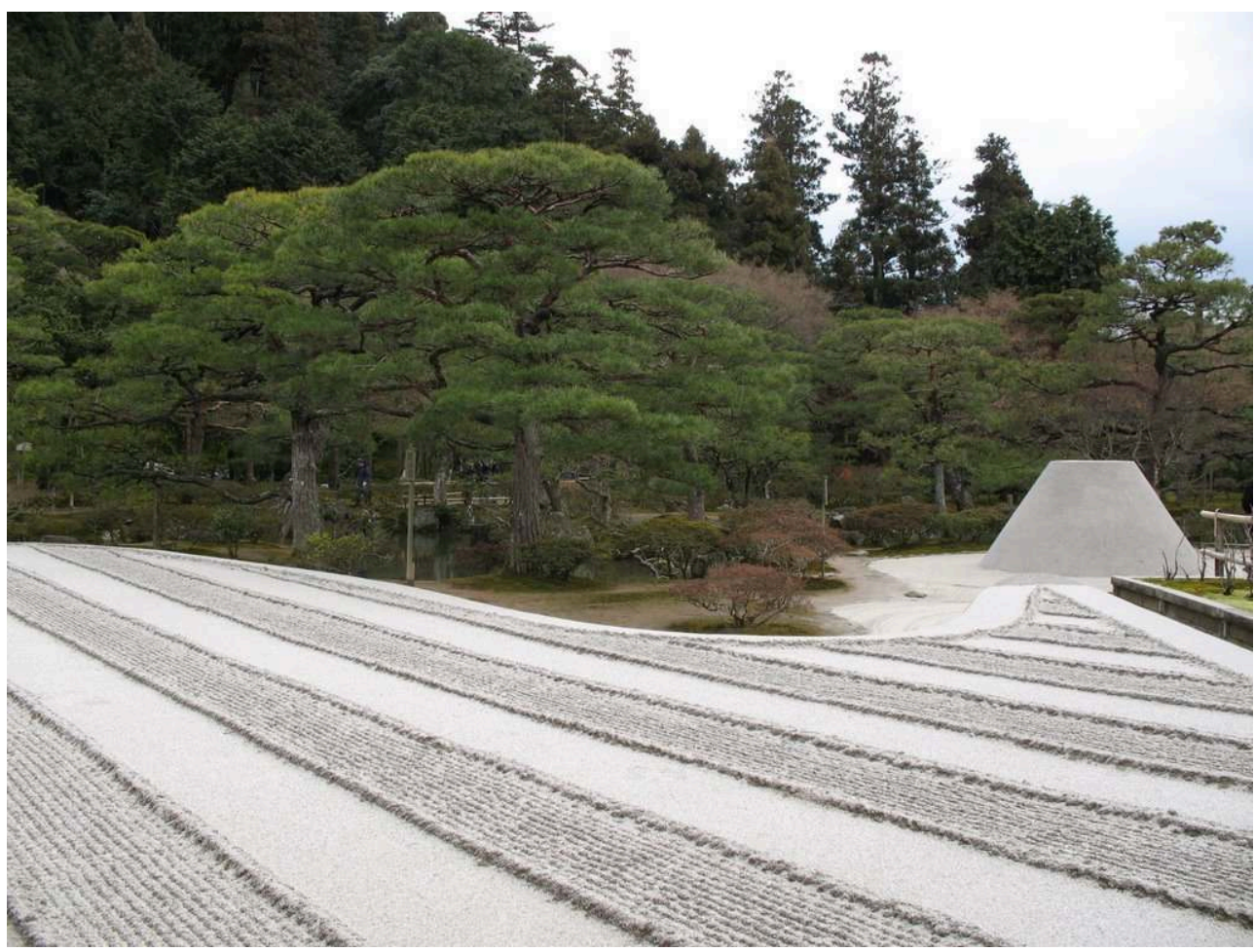

Figure 7. Le jardin Ryoan-ji

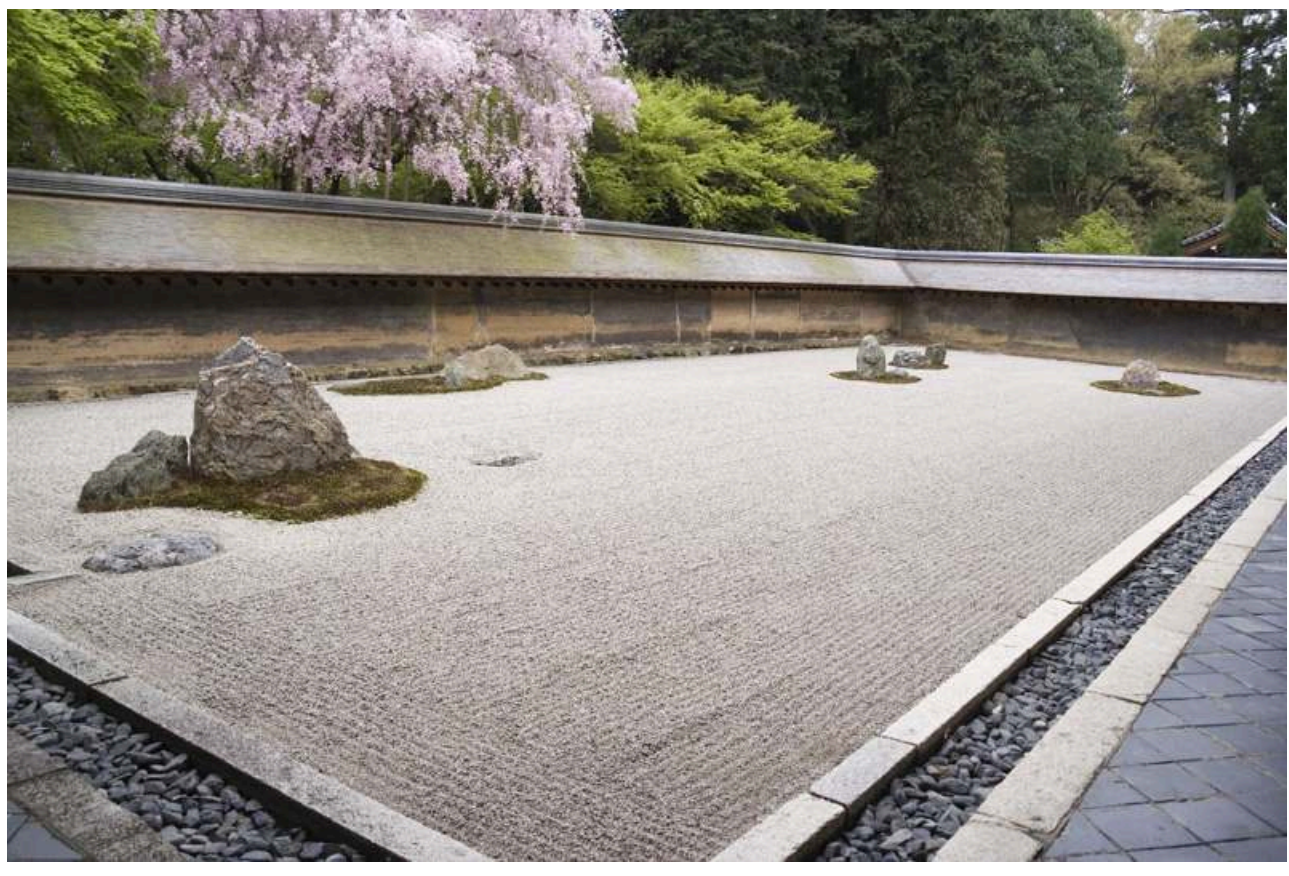

18 On ne peut généralement parcourir ces jardins, si ce n'est du regard qui peut se déplacer en eux ou s'y poser à partir de points différents offerts par la galerie qui borde le temple et donne ouverture sur eux. Dans l'un, une aire de gravier soigneusement ratissée et quelques rochers qui en émergent, dans l'autre une plage de sable régulièrement peignée en vaguelettes et le surgissement d'un cône tronqué en sable à la forme parfaite... et pourtant jamais d'en-face! Pourquoi? Parce que ces formes 
simples sont toujours en formation, non pas dans le sens où elles sont effectivement reformées chaque jour par un moine consciencieux, mais parce qu'elles sont toujours en formation d'elles-mêmes, dans l'acte de leur autogenèse, que peut accompagner le moine avec son râteau et le regard qui y est porté. Ainsi elles engagent celui qui les contemple à ne pas mettre en perspective le monde qu'elles constituent dans son regard, elles se refusent à devenir pour lui un objet, à déchoir en représentation, mais exigent une présence dans un état d'origine perpétuelle. Tel le koan qui brise la possibilité de saisie en représentation intellectuelle, les formes de ces jardins précipitent le regard de l'homme dans un nulle part en rompant avec toute forme figée par une analyse objectivante. Souvent confinés dans une aire physique restreinte, l'espace de ces paysages minéraux, ou simplement ces paysages - car en eux l'espace et le monde sont un - ouvrent à l'immensité du vide... éprouvé comme une plénitude enveloppante au milieu de laquelle nous sommes ici sans aucun système de référence, ni coordonnées ni point origine, mais à l'origine même de la présence surgissant de ce vide. Toutefois ce vide n'est pas béance mais patence. Son mouvement intérieur n'est pas d'engloutissement mais d'émergence : apparaitre qui se révèle dans son ouverture abrupte suivant une exigence contradictoire faite d'évidence et d'immotivation. Lorsque le regard parcourt les sillons rectilignes réguliers, les suit dans leurs contours ondulants qui encerclent les rochers, il participe au rythme qui est à l'œuvre dans leur autogenèse. La rencontre de ce paysage est celle du rythme même qui l'anime et qui se donne là, comme il a lieu, dans l'ordre du sentir in-formant, donnant forme à notre ressentir.

La compréhension, libre de toute confusion avec la notion de cadence, de ce qu'est authentiquement le rythme est indispensable à la création d'une œuvre paysagère, comme de toute œuvre réellement artistique, et en cela elle ouvre aussi à la révélation de la constitution de soi-même inhérente à celle du rythme. La mise en œuvre du rythme ne peut en aucun cas passer par l'usage du signe car, comme Henri Focillon l'a exprimé une fois pour toutes : «Le signe signifie, la forme se signifie. » (Henri Focillon, 1943, p. 10.) Henri Maldiney l'explicite lorsqu'il précise : «Une forme est son propre discours. En elle genèse, apparition, expression coïncident. Sa constitution est inséparable de sa manifestation et sa signification est une avec son apparaître. L'acte par lequel une forme se forme est aussi celui par lequel elle nous informe. Notre perception significative d'une forme n'a pas d'autre structure que sa formation. Cela veut dire qu'une forme s'explique elle-même. D'où - en nous limitant à sa dimension temporelle - le temps impliqué d'une forme ou d'un rythme générateur de formes coïncide avec son temps expliqué. » (Henri Maldiney, 2, 1973, p. 160-161.) Il en découle que le créateur - son œuvre naissant du rythme des formes qu'il découvre - et celui qui les rencontre sont, à l'identique, constitués dans et par le temps qui s'y déploie et qui ne peut être qu'un temps de présence. Temps de présence qui est nécessairement hors de celui de la représentation qui s'inscrit, elle, dans le temps quantifiable d'univers. L'intégralité de cette co-naissance s'accomplit dans un présent originaire. Ainsi «la relation du présent et du temps s'inverse. Le temps n'est plus au fondement du présent mais le présent au fondement du temps. » (Henri Maldiney, 2, 1973, p. 161.)

Concrètement, pour faire œuvre paysagère il faut des éléments fondateurs, des matériaux, à partir desquels l'artiste fait exister une forme. Il ne peut recourir pour cela à une formule, à un répertoire, ni même à un modèle qui relèvent tous d'une mise à distance objectivante dans l'ordre de la représentation. Seule sa rencontre avec le matériau donne naissance à une forme, en tant que rythme de ce matériau qu'elle 
trans-forme en lui permettant de la sorte d'accéder à une existence inédite. "Le rythme n'est pas de l'ordre des éléments fondateurs. Mais il n'est rien sans eux. Ni eux sans lui ne sont des éléments rythmiques. Il les transcende à travers eux. Supprimez leur résistance, il se dissipe. » (Henri Maldiney, 2, 1973, p. 163.) Le rapport du rythme et de ses éléments fondateurs constitue la question même du style, qui reste ouverte pour l'artiste.

\section{BIBLIOGRAPHIE}

Charcosset, J.-P. et Rordorf, B. « Présentation » dans Maldiney, H. Regard Parole Espace Lausanne, L'Âge d'homme, 1973.

Cheng, F., Shitao 1642-1707. La Saveur du monde, Phébus, Paris, 1998.

Focillon, H., La Vie des formes, Paris, P.U.F., coll. « Quadrige », 1943.

Maldiney, H., 1, « Le dévoilement de la dimension esthétique dans la phénoménologie d'Erwin Straus », Regard Parole Espace, Lausanne, L'Âge d'homme, 1973.

Maldiney, H., 2, « L'esthétique des rythmes » Regard Parole Espace, Lausanne, L'Âge d'homme, 1973.

Straus, E., Le Sens des sens (Vom Sinn der Sinne), traduit de l'allemand par G. Thinès et J.-P. Legrand, Grenoble, Éditions Jérôme Million, 1989.

\section{NOTES}

1. Traduit et cité par François Cheng dans Shitao 1642-1707. La Saveur du monde, Paris, Phébus, Paris, 1998, p. 46.

2. Huang Shan: la montagne jaune située dans le massif montagneux de l'Anhui méridional, province de l'Est de la Chine.

3. Omei Shan ou Emei Shan, montagne située dans le Sichuan à l'Ouest de la Chine, aux portes du Tibet.

4. Comme toujours fidèle à l'intransigeance que Maldiney maintient à l'égard de sa propre pensée, il donne à de nombreuses reprises des formulations différentes de ce constat dont celleci : « Le Réel c'est ce qu'on attendait pas - et qui toujours pourtant est toujours déjà là. », Regard Parole Espace, ibid., p. 152.

5. Straus, E., Le Sens des sens (Vom Sinn der Sinne), traduit de l'allemand par G. Thinès et J.-P. Legrand, Grenoble, Éditions Jérôme Million, 1989.

6. Straus, E., Vom Sinn der Sinne, p. 336, traduit et cité par H. Maldiney dans Regard Parole Espace, Lausanne, L'Âge d'homme, 1973, p. 143.

7. À propos de ce passage, Maldiney écrit: «... il faut se défaire d'une illusion théorique, de l'illusion théorique qui consiste à croire que toute l'expérience humaine est structurée par la polarité sujet-objet. La relation d'un sujet qui s'objecte le monde, et qui se distingue par là même de ce monde, n'est pas niable. Mais il s'agit là d'une situation seconde par rapport à la situation 
première qu'est la situation sensible. La relation Moi-Monde dans le sentir n'est pas réductible au rapport Sujet-Objet. » Ibid., p. 164.

8. Heidegger, M., «La spatialité de l'être-au-monde », Être et Temps, traduit de l'allemand par E. Martineau, édition numérique hors commerce, 1985, § 23.

9. Dhaulagiri, sommet des monts Annapurna à l'Ouest du Népal.

10. Créer son jardin pour le particulier japonais signifie y révéler l'intimité des moments à l'origine de sa propre présence. Inviter une autre personne dans son jardin implique de partager avec elle ces instants premiers de son ex-istence personnelle, ce que l'on appelle les existentiaux à partir desquels on devient soi-même. Il s'agit donc d'une invite peu commune et fortement significative.

11. Parmi les innombrables jardins du Japon, le visiteur pressé trouvera à Kyōto les paradigmes des deux formes évoquées dans cet article en se rendant à la villa impériale Katsura (Katsura Rikyū) et la villa impériale Shugaku-in.

12. De même que ethos en grec qui ne veut pas seulement dire manière d'être mais séjour, d'où la filiation entre l'esthétique et l'éthique.

13. L'occasion d'une journée de soleil après plusieurs jours de neige a mérité un long et pénible déplacement pour se rendre de Nagoya au temple Onodera, dans la préfecture de Mie, dont le jardin est d'habitude prisé pour ses pivoines, mais qui cette fois était recherché pour le paysage exceptionnel qu'il offre sous la neige illuminée par le soleil. Quoi que magnifié par la blancheur éclatante de la neige ensoleillée, il était frappant de constater le recueillement de ma jeune guide japonaise sans doute plus en prise avec l'étonnement de sa propre présence dans ce paysage qu'avec ma fébrilité reconnaissante à son égard pour m'avoir convaincu de subir ce long transport en vue d'une excursion pour moi uniquement touristique. Sans doute serais-je resté dans cette extériorité jubilatoire sans son calme qui a fait renvoi à mon appréciation objective et ouvert la porte à une soudaine extase esthétique, enfin partagée avec elle.

14. Cette correspondance entre humeur personnelle et atmosphère du milieu est précisément étudiée par Heidegger dans Être et temps sous le terme de Stimmung, diversement traduit par ambiance, tonalité affective, etc., le verbe stimmen signifiant en allemand "accorder", par exemple les cordes d'un instrument. En japonais, la parenté entre les deux dimensions se trouve dans le caractère commun employé signifiant l'énergie ou le souffle vital ( $k i)$, présent dans kibun, littéralement ma part du ki : l'humeur, la disposition personnelle, et fun'iki: l'atmosphère ambiante.

15. Toujours à Kyōto, deux jardins zen sont particulièrement remarquables et susceptibles de faire éprouver une rencontre de paysage présentée là dans sa forme la plus épurée : le Ryōan-ji et le Ginkaku-ji (temple du Pavillon d'argent situé à l'est de la ville à ne pas confondre avec le Kinkaku-ji, temple du Pavillon d'or situé lui à l'ouest de la ville).

\section{RÉSUMÉS}

Ce que l'on appelle « paysage » est le moment où un milieu apparaît à un individu. Il suppose leur rencontre. Celle-ci repose sur une capacité d'étonnement qui ouvre à un rythme commun aux deux, celui de l'unicité originaire d'où ils existent leur être propre. Ce processus n'est possible qu'en rupture avec la représentation de l'environnement habituel qui constitue en objets séparés le monde et le sujet. C'est dans le passage du sentir au ressentir que sujet et monde retrouvent leur union constitutive primordiale qui apparaît là comme paysage. Ce texte tente de mettre en 
prise avec cette situation première de chaque instant en explicitant des exemples naturels et artificiels de paysages. Il a pour but de contribuer à la création de tels instants par les paysagistes.

What we call 'landscape' is the instant when a milieu appears to an individual. It assumes their meeting. This one rests on a capacity of astonishment which opens to a rhythm common to both, that of the original uniqueness from where they exist their own being. This process is possible only in break with the presentation of the habitual environment which constitutes in separated ob-jects the world and subject. It is in the transition from sensation to feeling that the subject and the world find their primordial constituting union which appears there as landscape. This text tries to engage with this primeval situation of every moment by clarifying examples of natural and artificial landscapes. It is aimed at contributing to the creation of such instants by the landscapists.

\section{INDEX}

Mots-clés : étonnement, rythme, unicité, rencontre, paysage

Keywords : astonishment, rhythm, oneness, meeting, landscape

\section{AUTEUR}

\section{JOËL BOUDERLIQUE}

Joël Bouderlique est philosophe et psychologue, ancien professeur d'université au Japon et chercheur indépendant et traducteur du Pr. Bin Kimura.

joel.bouderlique[at]free[dot]fr 